FERMILAB-Conf-89/79

\title{
Radiative Polarization in High-Energy Storage Rings *
}

\author{
S. R. Mane \\ Fermi National Accelerator Laboratory \\ P.O. Box 500, Batavia, Illinois 60510
}

March 1989

* Presented at the 1989 IEEE Particle Accelerator Conference, Chicago, Illinois, March 20-23, 1989.

늘 Operated by Universitles Research Association, Inc., under contract with the United States Department of Energy 


\title{
RADIATIVE POLARIZATION IN HIGH-ENERGY STORAGE RINGS
}

\author{
S.R. Mane \\ Fermi Natjonal Accelerator Laboratory, ${ }^{* P . O . ~ B o x ~ 500, ~ B a t a v i a, ~ I L ~} 60510$
}

\begin{abstract}
Fleciron and pusit rul beams circulating in high-energy atorage rings berome spontanmusly polarized by the emission of aynchrotron radialion. The asvmpiolic degree of polarization that can be attained is st.rongly affect of by sn-called depolarizing resonances. Detailed experimental measurements of the polarization were made SPEAR about ten years ago, but due to lack of a suitable theory only a limited theoretical fit to the data has so far been achieved. I present a general formalism for calculating depolarizing resonances, which has been coded into a compules program called SMILE, and use it to fit the SPEAR data. Bv the use of suitable approximations, I am able to fit both higher order and nonlinear resonances, and thereby to interpret many hitherto ulexplained features in the data, and to resolve a puzzle concerning the asvmmetry of certain resonance widths seen in the data.
\end{abstract}

\section{Introduction}

It was predicted by Sokolov and Ternov [1] that electrons and positrons circulating in high energy storage rings would become polarized by the emissinn of synehrotron radiation; this effect is now ralled the "Snknlov-Ternov effect." They solved the Dirac equation in a homogenous vertical magnetic field and predicted an asymptotic degree of polarizat ion of $8 /(5 \sqrt{3}) \simeq 92.4 \%$. In practice, the polarization is sometimes reduced from this value by so-called "depolarizing spin resonances." A formula for the polarization including these resonances was derived by Derbenev and Kondratenko, [2] and is now known as the Derbencs-Kondratenko formula. Experimental measurements of the polarizatiun, showing several resonances, were made at the storage ring SPEAR $: 3$. Only the first order resonances in these data have been fitted; $\left[t_{1}\right.$ the widths of the rest have remained unexplained up to now. A formalism to calculate the widths of arbitrary spin resonances was given in Ref. [5], and a computer program called SMILE was written based on it. This program has been used to fit the SPEAR data, after making various approximations, and I have used it to explain various puzzling features seen in the data. Some of this work was presented in Ref. [6], and more details in Ref. [7], including new theoretical predictions which could serve as a check on the theory.

\section{General Remarks}

The Derbenev-Kondratenko formula is [2]

$$
P_{e q}=\frac{8}{5 \sqrt{3}} \frac{\left(\frac{1}{|\rho|^{3}} \hat{b} \cdot\left(\dot{n}-\gamma \frac{\theta \dot{n}}{\partial \gamma}\right)\right)}{\left\langle\frac{1}{|\rho|^{3}}\left(1-\frac{2}{9}(\dot{n} \cdot \dot{v})^{2}+\frac{11}{18}\left|\gamma \frac{\partial \dot{n}}{\partial \gamma}\right|^{2}\right)\right)},
$$

where $P_{e q}$ is the equilibrium degree of polarization, $\rho$ is the local radius of curvature of the particle trajectory, $\dot{v}$ is the direction of particle motion, $\vec{b}=\vec{v} \times \dot{\vec{v}} /|\vec{v} \times \dot{\vec{v}}|_{,} \dot{\boldsymbol{n}}$ is the spin quantization axis on $\mathrm{a}$ trajectory, and the angular brackets denote an ensemble average over the particle trajectories and accelerator aximuth. This formula was rederived, and the notation clarified, in Ref. [8]. The principal details are given in Rcf. [9]. The algorithm in Ref. [5] evaluates the vectors $\hat{n}$

\footnotetext{
Operated by the Universities Rewearch Associalion Inc., under contract with the U.S. Department of Energy
}

and $\gamma(\theta \hat{n} / \partial \gamma)$ using a perturbation expansion, to be described briefly below. The various spin resonances are obtained by systematically expanding the perturbation series. A copy of the data in Ref. [3] is shown in the inet to Fig. 1. Note that the curve therin is a guide to the eye, not a theoretical fit.

\section{Calculation of Resonances}

A brief description of the algorithm in Ref. [5], frequently called the "SMILE algorithm," will be given below. It trests all resonances, in principle, in the approximation of linear orbital dyanjics. A more general algorithm which also treats nonlinear orbital dynamics has been publikhed by Yokoya [10]. A number of more limited algorithms, which treat a subset of the resonances included in the above algorithms, are given in Rers. [11] - [15]. I have proved the algorithms in Refs. $[5,11,12,13,15]$ to be mathematically equivalent, when restricted to * common domain of approximation, in either Ref. [5] or [6]. Ref. [14] has been shown to be equivalent to the rest in Ref. [17]. The bove are all analytical algorithms. A numerical tracking algorithm has been presented in Ref. [16], which also treats arbitrary tesonances.

I now restrict attention to the SMILE algorithm [5]. I rinote the accelerator aximuth by $\theta$. The equation of motion lor $\hat{n}$ is

$$
\frac{d \hat{n}}{d \theta}=\overrightarrow{\mathbf{n}} \times \hat{n},
$$

where $\vec{\Omega}$ is the spin precestion vector of the storage ring [18]. I decompose $\overrightarrow{\boldsymbol{\Omega}}=\vec{\Omega}_{0}+\overrightarrow{\boldsymbol{w}}$, where $\vec{\Omega}_{0}$ is the value of $\overrightarrow{\boldsymbol{\Omega}}$ on the accelerator closed orbit, and $\vec{\omega}$ describes the additional terms due to orbital oscillations around the closed orbit. Let $\dot{n}_{0}$ denote the value of $\hat{n}$ on the closed orbit, and let $i_{0}$ and $m_{0}$ denote the two other linearly independent $10-$ lutions of Eq. 2 on the closed orbit: $d f_{0} / d \theta=\vec{n}_{0} \times \hat{l}_{0}$, etc. Numerical colution of Eq. 2 for $\left\{\hat{l}_{0}, \dot{m}_{0}, \hat{n}_{0}\right\}$ is by now standard.

I now write $\dot{n}=n_{1} \hat{l}_{0}+n_{2} \hat{m}_{0}+n_{3} \hat{n}_{0}$, and define spherical harmonics via $V_{ \pm 1}=\mp\left(n_{1} \pm i n_{2}\right) / \sqrt{2}, V_{0}=n_{3}$. The equation of motion for $V_{ \pm 1}$ and $V_{0}$ is

$$
\frac{d}{d \theta}\left(\begin{array}{l}
V_{1} \\
V_{0} \\
V_{-1}
\end{array}\right)=\dot{\omega} \cdot J^{T}\left(\begin{array}{l}
V_{1} \\
V_{0} \\
V_{-1}
\end{array}\right) .
$$

The formal wolution is [5]

$$
\left(\begin{array}{l}
V_{1} \\
V_{0} \\
V_{-1}
\end{array}\right)=T\left\{\exp \left(\int_{-\infty}^{0} i \vec{\omega} . J^{T} d \theta^{\prime}\right)\right\}\left(\begin{array}{l}
0 \\
1 \\
0
\end{array}\right) .
$$

The symbol "T" denotes a time-ordered, or $\theta$-ordered, product. To obtain a practical solution, I expand the exponential in \& power series, and evaluate the resulting integraln term by term: thit is the SMILE perturbation series [5].

\section{Fit to Data}

A theoretical fit to the SPEAR date [3] is shown in Fig. 1. It was produced uning the SMILE program [5]. The details of the fit are given in Ref. [7]. The locations of resonances are given by

$$
v=k_{0}+k_{1} \nu_{z}+k_{2} \nu_{v}+k_{3} \nu_{3} .
$$




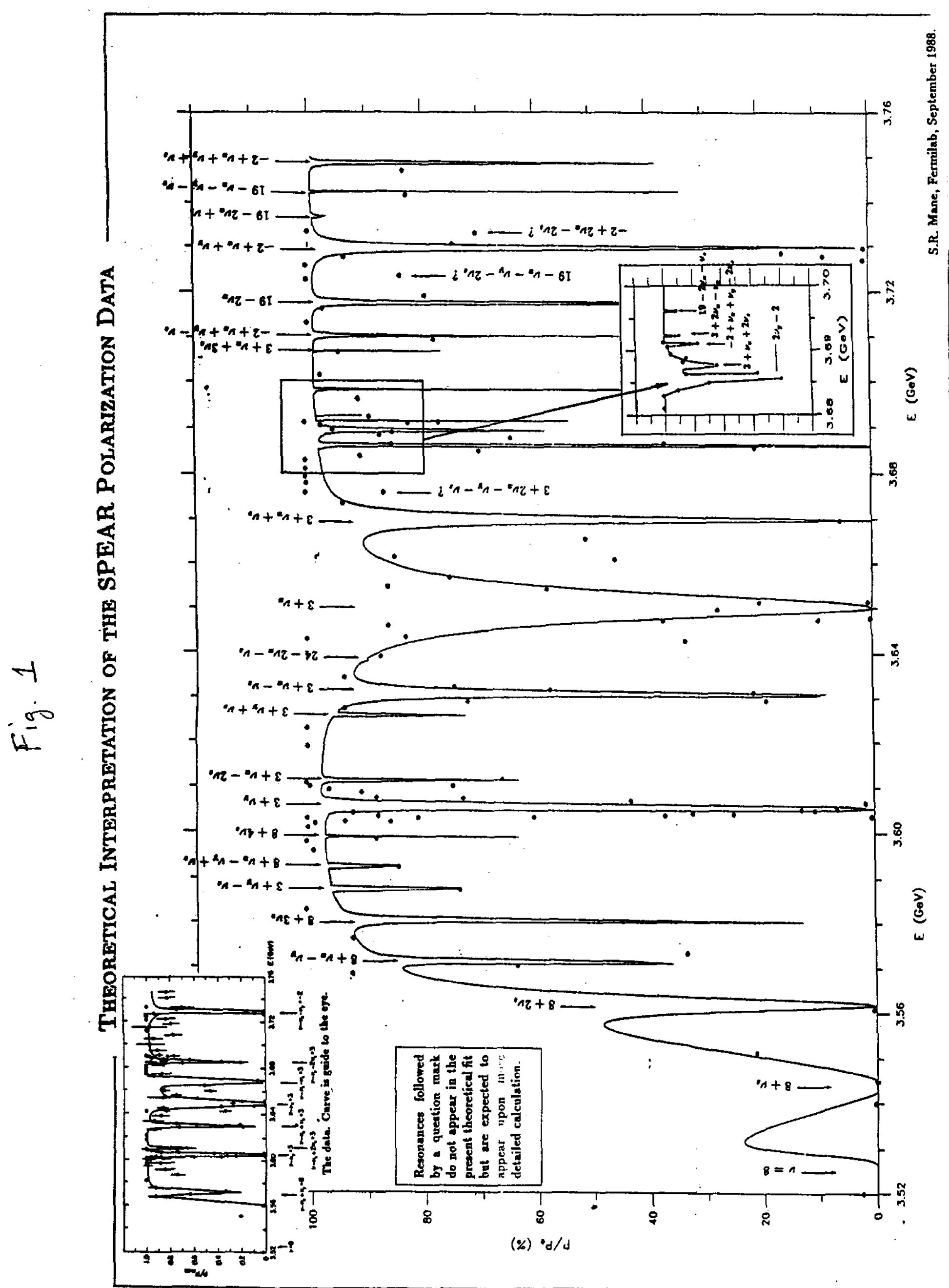


llere $\nu$ is the spin tuice and $\nu_{i}, i=\Sigma, y_{1}, w_{1}$ are the orbital tunes. The $t_{i}$ are integers. including zero. Various resonances are identified by the experimenters in the inset to Fig. 1 [3]. The tunes were read off from il, Hisl used in the theortical fit. In fact two sets of tunes wre used, aljote nind below $3.60 \mathrm{GeV}$, because the tunes were not constant thronghou! the whole of Fig. 1. Another important fect to note is thut the scsonance widths depend on imperfections in the nechine, so $n$ srt of distorted elosed orbits was produced by using different raninm seeds, and the sum total of these results was used to prepare the fil. This is an important reason why prediction of absolute resonance withihs is very difficult in general. $\boldsymbol{A}$ simple closed orbit smoothing proseduse was used, which consisted of suppressing the Fouries hartinnics of the closed orbit closest to the vertical betatron lune $\left(\nu_{y} \simeq 5.18\right)$. The global seale of the random kicks was choen so as to approximately fit the resonance $\nu=3+\nu_{\text {a }}$ at $3.65 \mathrm{GeV}$. The resulting $t . \mathrm{m} . \mathrm{s}$. closed orbit amplttude varied from 0.6 to $1.2 \mathrm{~mm}$. In addition, the r.m.s. verlical betatron tunespread was sead off from nnother graph in Ref. [3]. This was attributed to traniverse nonlinear 1 unespread, alid a simple model of tunespread was introduced to fit lie nonlinear resonatices $\nu=3+v_{y}$ at $3.605 \mathrm{GeV}$ and $\nu=2 v_{y}-2$ at $3.086 \mathrm{Gcl}$. A fict the inachine parameters had been fixed in this way, all the resnumaces werc calculated without further modifications.

Although a lol of the input for the theoretical fit involved guess. work, besause after ncarly ten years detailed information was difficult to find, it is still possible explain some of the puzzling features in the experimental data. Note that the experimental curve in Fig. I is a guide to the cyc, not a theoretical fit. Some depolatizing res. nllanees are iricnlificd. The analysis in Fig. 1 reveals, in addition, the existence of several more narrow resonances in the data, thowing that the experimental measurements were very precise. It appears that datn ignored as st atistical noise experimentally actually were real physics. In particular, one theoretical prediction in that the resonances $\nu=3+v_{x} \pm 2 \nu_{3}$, at $3.61 \mathrm{GeV}$ and $3.69 \mathrm{GeV}$, should have approximately equal width. Here $\nu$ is the apin tune and $\nu_{i}, i=x, y_{1}$, , are the orbital l incs. It can be clearly scen that the two resonances above have very unequal widths. By using SMILE, I am able to show that the data around $3.69 \mathrm{Gil}$, drawn as one wide resonance in the inset to Fig. 1 , in lact consisis of several narrow, nearly overlappling resonances. The tesonances $v:-3+v_{z} \pm 2 v$, do in fact appear to huve epproximately cyual width, after the theoretical analysis. A more detailed fit to the data in the virisity of $3.09 \mathrm{GeV}$ is shown in Fig. 2.

Further delaik are given in Rel. [7]. In addition, the above reference a)so contains some theoretical predictions about the behavior of the resonance widtlis, which should be experimentally teutable.

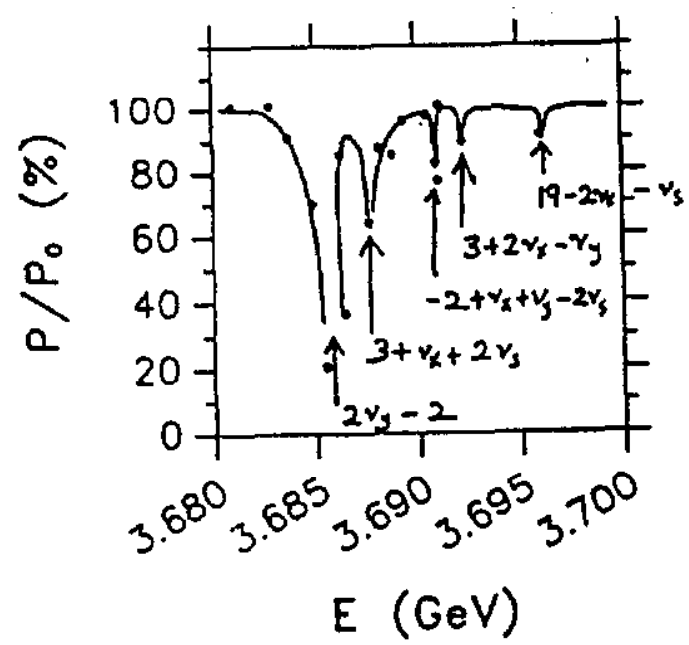

Fig. 2 Resonance spectrum in the region around $3.69 \mathrm{GeV}$.

\section{Acknowledgements}

This work was supporled by the Univerities Research Association Inc., under Contract DE-AC02-76CH03000 from the Department of Energy.

\section{References}

\section{References}

(1) A.A. Sokuluv and I.AT. Ternov, Dokl. Akad. Aked. Nauk SSSR 153, 1052 (1963) [Sov. Phys. Doklady 8, 1203 (1964)].

[2] Ya. S. Duthenev nnt A.M. Kondratenko, Zh. Eksp. Teor. Fiz. 64, 1918 (197:1) [Sov. I'hys. JETP 37, 968 (1973)].

(3) J.R. Jolusson ef al., Nuel. Instrum, Meth. 204, 261 (1983).

[4] A. Choo, Procceding: of the 1980 International Symposium on High-Energy Spin Physies with Polarized Beams and Polarized Targets, Tnusanne, Switzerland, September 1980.

[5] S.R. Mane, Phys. Rev. A 36, 120, (1987).

[6] S.R. Mane, "Higher Order Spin Resonances," presented at the $8^{\text {th }}$ International Symposium on High Energy Spin Phyict, Minneapolit, Minnesote, Sept. $12-17,1988$.

[7] S.R. Mane, Fermilab FN.503 (1889).

(8) S.R. Mane, Phys. Rev. A 36, 105, (1887).

[9] S.R. Mare, Phys. Rev. Lett. 57, 78 (1986).

[10] K. Yokoya, Nud. Instrum. Meth. A258, 149 (1987).

[11] A. Chro, Nucl. Instrum. Meth. 28, 180 (1981).

[12] K. Yokoya, Partiele Aecelerators 13, 85 (1983).

[13] C. Biscari et al, I Nuovo Cimento 81, 128 (1984).

[14] L.N. Fand and A. Skuja, Phys. Rev. Lett. 691910 (1987).

[15] J. Buon, "A Stochastic Model of Depolarization Enhancement due to Large Energy Spread in Electron Storage Rings," presented at the $8^{\text {th }}$ International Sympotium on High Energy Spin Physics, Minneapolis, Minnesota, Sept. 12-17, 1988.

[16] J. Kewisch et 2l., Phys. Rev. Lett. 62, 419 (1989).

(17) J. Buon, Nuel. Instrum. Meth. A275, 219 (1989).

[18] Ya.S. Derbenev and A.M. Kondratenko, Zh. Eksp. Teor. Fiz. 62, 430 (1972) [Sov. Phys. JETP 35, 230 (1872)]. 